\title{
Meat grinder hand injuries: serial cases
}

\begin{abstract}
Meat grinder injuries to the hand are not common but it is proved to be very mutilating. Meat grinder injury causes a wide spectrum of injuries that can bring a physical and emotional pain in addition to the economical impact on the life the individuals.

Here three cases of meat grinder hand injuries are presented. Two were children and one was adult. The children came while their hand was still caught in the meat grinder but the adult was first seen in a very big university cafeteria meat grinder.

It is important to recognize the severity of these types of injuries to the patient and to the society in which the patient lives. Prevention by increasing awareness and following safety measures should given priority. But when it happens gently extrication of the hand from the grinder without causing further injury should be practice together with possible reconstruction.
\end{abstract}

Volume 6 Issue 6 - 2017

\section{Berhe Gebreslassie Kassa}

Department of Orthopedics and Trauma Surgery, Mekelle University, Ethiopia

Correspondence: Berhe Gebreslassie Kassa, Department of Orthopedics and Trauma Surgery, Orthopedic Surgeon, School of Medicine, College of Health Sciences, Mekelle University, Ethiopia,Tel +2519|4367429, Email berhe5907@yahoo.com

Received: May 16,2017 | Published: May 23, 2017

Keywords: hand injury, meat grinder, fractures, sparing

\section{Introduction}

Mutilating hand injuries are commonly seen in industrial accidents. Of the cases, negligence and inexperience are found to be the chief predisposing factors. ${ }^{1}$ Meat grinder hand injuries are one of the causes that can give mutilating hand injury even though it is not common. It causes a wide spectrum of injuries that result in tremendous physical and emotional pain in addition to significant economic impact. ${ }^{2}$ When it occurs in children the impact is also life time touching pain to the family. These days, the use of meat grinders is increasing because of the growth income in a country without having enough awareness on its complications. So here we present case series of three meat grinder hand injuries and their outcomes.

\section{Case I}

On April 17, 2015 there was a call to our department from our main campus of Mekelle University where there was a 24-year-old male patient who was caught his right hand by a big meat grinder of the students cafeteria. When we reach there he was in severe pain and his hand was still in the grinder. We gave him strong analgesics. Trial of breakage of the meat grinder by different types of drills and saws was done but it was not possible. After communication, reversal of the grinder with adjustable wrench done and the hand was easily removed after staying for two hours and half in the grinder. Immediately, the patient was taken to Ayder referral hospital operation theater, there was severely crashed hand for which fixation of the fractures and approximation of the wound done. After following the patient for 3 weeks with antibiotics, analgesics, and daily wound care patient had second surgery which was amputation of the $2^{\text {nd }}$ to $4^{\text {th }}$. Digits and partial amputation of the $1^{\text {st }} \& 5^{\text {th }}$ digits.

\section{Case 2}

A 4-year-old male patient was relatively well 20 minutes back at which time he put his right hand on meat grinder which was turned on \& grinding meet. The family saw what happed after the child screamed \& turned the grinder off. The family could not free the child's hand out of the grinder because it was stack in it. There was no bleeding noticed. He was brought to the hospital with his right hand stack in the meat grinder. Figure $1 \mathrm{He}$ was taken to the operating theatre and under general anesthesia; the grinder was reversed with the wrench until the hand was released. The $3^{\text {rd }}$ and $4^{\text {th }}$ fingers were severely crashed and we did immediate amputation and the first was not injured. $2^{\text {nd }}$ and $5^{\text {th }}$ were fixed by k-wire and wound approximated but after 2 weeks the digits became gangrenous and for which we did amputation (Figure 2).

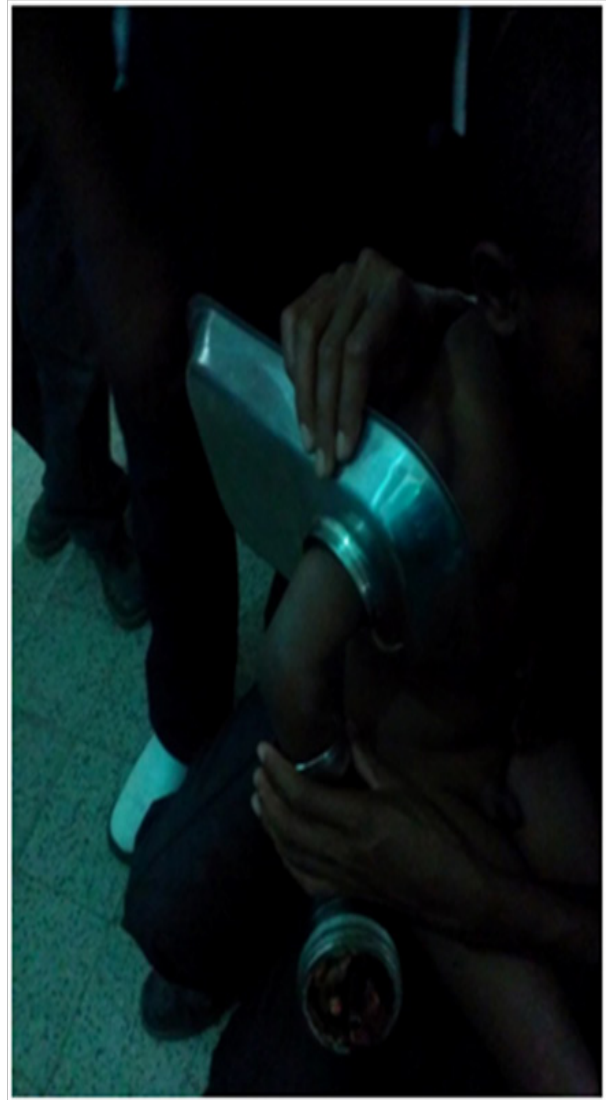

Figure I crashed hand injury before removal from a meat grinder. 


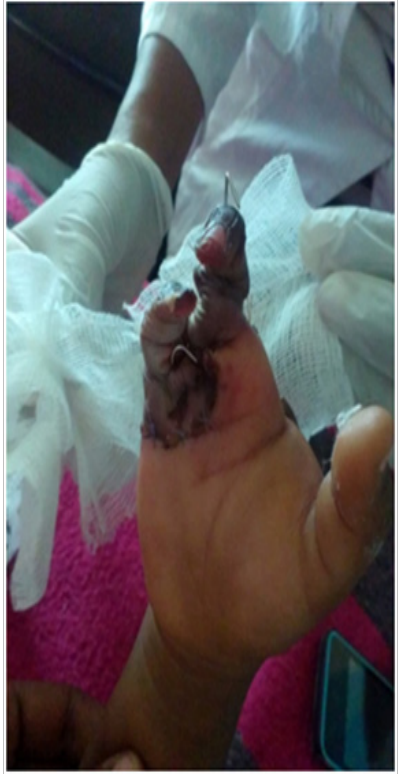

Figure 2 Crashed hand after removal from a meat grinder and fixed by $\mathrm{K}$-wire in the ward.

\section{Case 3}

A 2-year-old female child presented by her family on September 13, 2015 30minutes after her hand was caught by meat grinder. She inserted her right hand suddenly while it was working. Her family turned off and brought her while the hand was still inside the grinder. Figure 3 She was taken immediately to the operation theater and under general anesthesia; the grinder was reversed with a Rongeur until the hand was released. Figure $4-6$ The $2^{\text {nd }}$ to $5^{\text {th }}$ fingers were severely crashed, fractured bone fixation and wound approximation together with antibiotics and analgesics tried but after 9days the digits became gangrenous and for which we did amputation. The 1st finger was good.

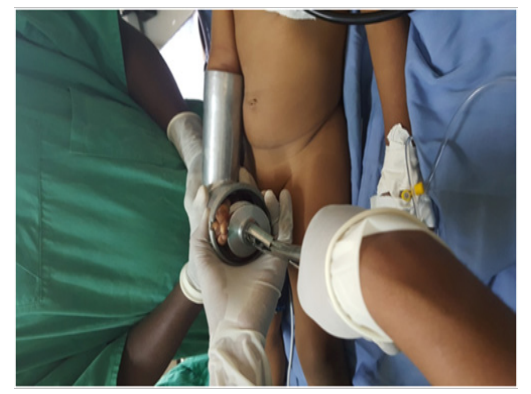

Figure 3 Crashed hand injury before removal from meat grinder machine.

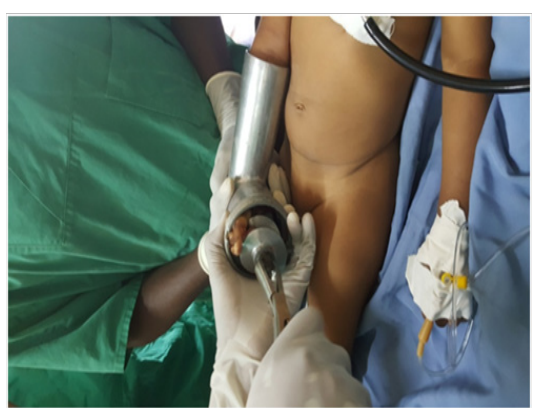

Figure 4 Crashed hand injury during reverse (anti clock wise) removal of the feed screw from head tube of meat grinder.
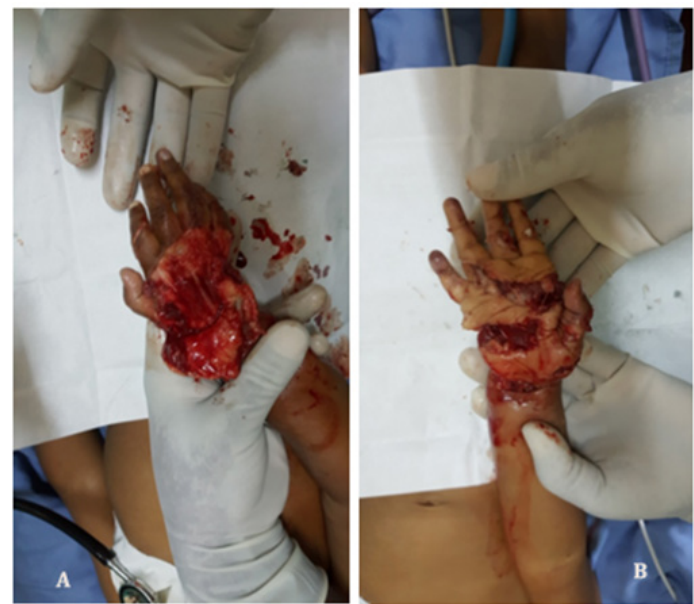

Figure 5A \& 5B Anterior \& posterior aspect of crashed hand injury after removal from meat grinder respectively.

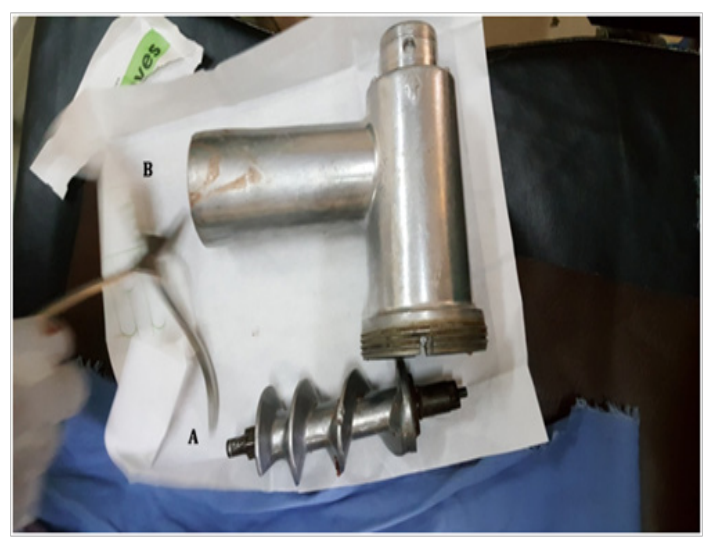

Figure 6 Meat grinder components which are related with hand: a. head tube \& b. feed screw.

\section{Discussion}

Meat grinder injuries to the hand are not common but when they occur they are very mutilating. Our patients were one adult and two children which indicated that it can happen in any age group. In all cases there was involvement of the right dominant hand which can be explained by the involvement of their right hand in the majority of function; which is going to have a big negative impact in the functions of the dominant hand and the economical impact on the life of the individuals especially to the adult one who requires long duration to change his handedness in addition to physical and emotional pain of all our patients.

The most important concept of management of these types of injuries is careful removal of the caught hand from the grinder without causing additional injuries but before taking the patient to the operation theater it is important to know how to operate the grinder machine so that we will not spend any time to search or try for it. After preparation and under general anesthesia or sedation or strong analgesics like in our case 1 where you cannot transport the patient together with the grinder, each hand was released from the grinder by reversed turning of the grinder with adjustable wrench or Rongeur until the injured hand was completely free. Following extrication of the injured hand from the grinder, we must restore the structure and function of the hand, required debridement with preservation of viable tissues, irrigation of the wound and the use of antibiotics and 
analgesics. We have noticed also that duration presentation has an effect on the outcome like sparing the thumbs in both cases $(2 \& 3)$ who presented with short duration.

But the setup in our case, there is no plastic or hand surgeon who can help these patients better and we tried fixation and closure of the wound after debridement and the result was sparing only either the thumb or part of it. So the concept of prevention for this type of injuries even becomes more important when you do not have enough and skilled full staff members like ours or similar setups.

So it is important to recognize the severity of these types of injuries to the patient and to the society in which the patient lives. Prevention by increasing awareness and following safety measures should be given priority. But when it happens gently extrication of the hand from the grinder without causing further injury should be practice together with possible reconstruction.

\section{Acknowledgements}

To Dr. Danayt Kahsay, my resident, who helped me in getting the history and pictures of one of my three patients. To Ayder comprehensive specialized hospital, Mekelle University for providing the facility.

\section{Conflict of interest}

The author declares no conflict of interest.

\section{References}

1. Blair SJ, Allard KM. Prevention of trauma: a cooperative effort. $J$ Hand Surg. 1983;8(5):649-654.

2. Brandner M, Bunkis J, Trengove GT. Meat grinder injuries to the upper extremity. Annals Plast Surg. 1985;14(5):454-456. 